\title{
Signal-to-noise ratio of nonlinearity recorded holograms of diffuse objects
}

\author{
A. Fimia, A. Beléndez, and L. Carretero
}

\begin{abstract}
A nonlinear model for holographic recording materials is used to evaluate the signal-to-noise ratio in diffuse-object holograms. A comparison of this model with the linear model proposed by Upatnieks and Leonard [J. Opt. Soc. Am. 60, 297 (1970)] shows that our model justifies the experimental results obtained for dieletric holograms at high density before bleaching.

Key words: Holographic recording materials, diffuse-object holograms, nonlinearities.
\end{abstract}

\section{Introduction}

Intermodulation noise has been considered the most important source of noise when the signal of diffuse objects is stored holographically. In 1970, Upatnieks and Leonard proposed a linear model in which they predicted the signal noise for dielectric diffuseobject holograms. ${ }^{1}$ A recent study on the sources of noise in interconnection holographic systems, in which the interference of multiple beams on a reference beam was analyzed, also accepted the hypothesis that the behavior of the recording material was linear and found that there was no agreement between the models of multiple and reference beams for diffuse objects in which intermodulation noise was found. ${ }^{2}$ In this paper we use a Upatnieks and Leonard modified model to analyze the signal noise of diffuse objects when the behavior of the index variation of the recording material is not linear in relation to exposure.

Previous studies have shown that the behavior of the signal-to-noise ratio (SNR) in diffuse-object holograms is different from the behavior proposed in the literature when bleached emulsions are used. In this case, high values of SNR are achieved at high exposures, and it is specifically in the nonlinear zone of the developer that the highest values are achieved and maximum diffraction efficiency is reached. ${ }^{3}$

The authors are with the Universidad de Alicante, Apartado 99, Alicante E 03080, Spain; A. Fimia and L. Carretero are with the Laboratorio de Optica, and A. Beléndez is with the Departamento de Ingeniéra de Sistemas y Comunicaciones.

Received 25 January 1994; revised manuscript received 23 May 1994.

0003-6935/94/327606-05\$06.00/0.

(1) 1994 Optical Society of America.

\section{Theory}

If we assume that the variation in density is reached during developing, ${ }^{4}$ we can see that the variation in the index of refraction for bleached holograms is given by

$$
\Delta n=\Delta n_{0}[1-\exp (-\beta E)],
$$

in which $\Delta n_{0}$ is the maximum modulation index that is reached and $\beta$ is a parameter specific to the process itself that depends on the sensitivity of the developer. (If we take the first two terms of the series expansion of the exponential function, we find $\Delta n=\Delta n_{0} \beta E$, and the linear behavior proposed in Ref. 1 is once again achieved.)

If we substitute Eq. (1) into Eq. (6) of Ref. 1, which claims that the index modulation associated with the intermodulation noise is not linear, we find that the normalized unscattered fraction of the light amplitude is given by

$$
A=\exp \left(-i \mu \Delta n_{0}\right) \sum_{n=0}^{\infty} \frac{\left(i \mu \Delta n_{0}\right)^{n}}{n !} \frac{1}{1+2 \beta \tau \sigma^{2} n} .
$$

In Eq. (2), $\mu=2 \pi d /(\lambda \cos \theta)$, where $d$ is the thickness of the holographic recording material, $\lambda$ is the reconstruction wavelength, $\theta$ is the Bragg angle, $\tau$ is the exposure time, and $\sigma^{2}=a_{0}^{2} / 2 K$, where $K$ represents the ratio of the object to the reference beam intensities and $a_{0}$ is the amplitude of the reference wave that is incident upon the recording material.

By keeping in mind the definition of SNR given by Upatnieks and Leonard ${ }^{1}$ [SNR $=A A^{*} /\left(1-A A^{*}\right)$, where * represents the complex conjugate], and by taking into account that $\tau a_{0}^{2}=[K /(1+K)] E$, where $E$ is exposure time, we can obtain the SNR as a 
function of $E$ :

$$
\mathrm{SNR}=\frac{1-m^{2}\left(a-b^{2}-1 / 4 m^{2} a^{2}\right)}{m^{2}\left(a-b^{2}-1 / 4 m^{2} a^{2}\right)},
$$

where, in order to obtain Eq. (3), we take the first three terms of the series expansion of Eq. (2). In this equation, $a=(1+2 \xi E)^{-1}$ and $b=(1+\xi E)^{-1}$. The following variable changes are utilized: $\quad \xi=\beta(1+K)$ and $m=\mu \Delta n_{0}$. As can be seen, this function is clearly different from the one proposed by Upatnieks and Leonard. Moreover, we can see that the dependence of the SNR function can be expressed by parameters that depend on the characteristics of the recording medium $(m)$ and on the processing it is submitted to $(\beta)$. Figure 1 shows the signal noise function for different values of parameter $m$ and $\xi$. We can see that parameter $m$ is related to the minimal value of SNR, while parameter $\xi$ shows us its behavior at high exposures.

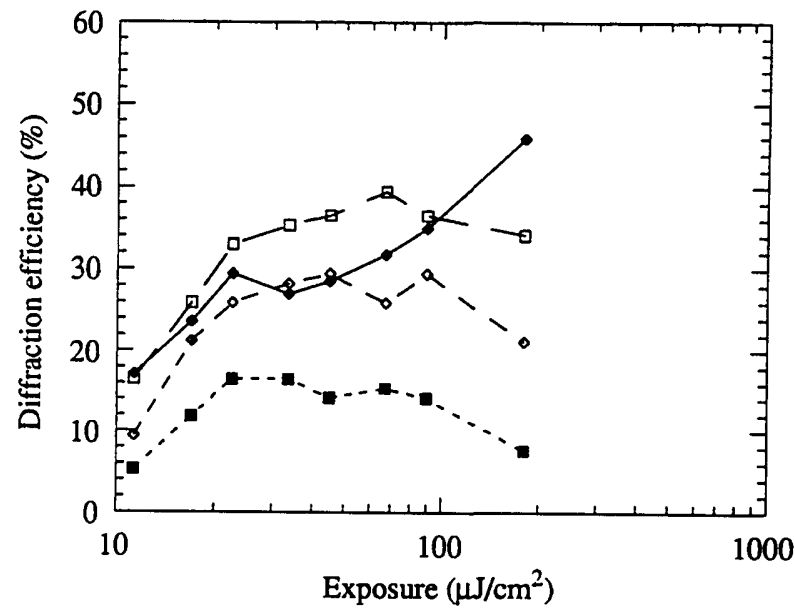

(a)

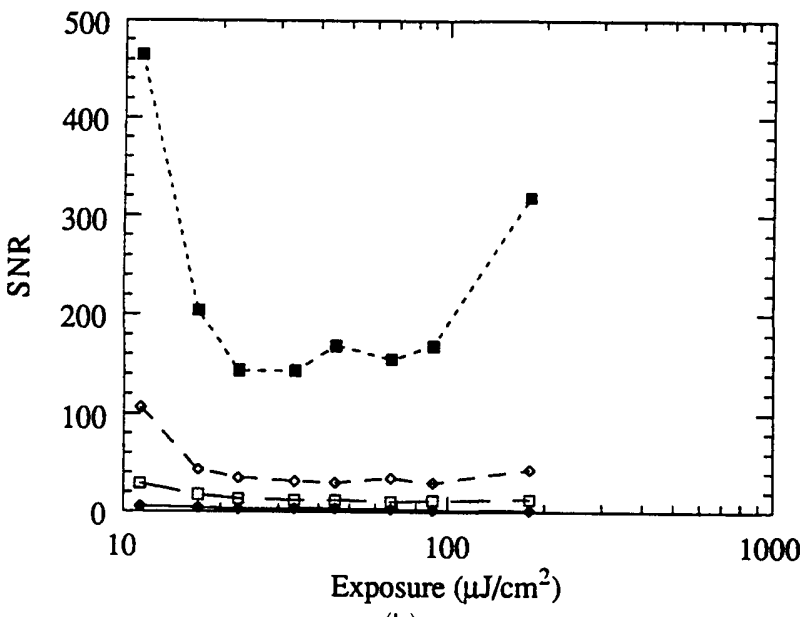

(b)

Fig. 1. (a) SNR as a function of exposure for $m=0.5$ and the following: $\xi=0.12$ (filled squares), $\xi=0.065$ (hollow squares), $\xi=$ 0.055 (filled diamonds), and $\xi=0.042$ (hollow diamonds). (b) SNR as a function of exposure for $\xi=0.065$ and the following: $m=$ 0.50 (hollow squares), $m=0.27$ (filled diamonds), $m=0.215$ (hollow diamonds), and $m=0.13$ (filled squares).

\section{Experimental Results and Discussion}

In order to compare the linear and nonlinear models, we recorded diffuse-object holograms in Agfa-Gevaert 8 E75 HD emulsion, varying the beam relation and the exposure time. The signal of a $2 \mathrm{~cm} \times 2 \mathrm{~cm}$ diffuse object with a central opaque zone measuring 1 $\mathrm{cm} \times 1 \mathrm{~cm}$ was stored so that the SNR could be measured as the ratio of light intensity in the dark zone to that in the illuminated zone when the real image was reconstructed. The reference beam was collimated and formed a $40^{\circ}$ angle to the normal to the surface of the photographic emulsion. A He-Ne laser $(\lambda=633 \mathrm{~nm})$ was used for both the storage and the reconstruction of the diffuse-object holograms. The processing used is shown in Table 1. The developer employed was D-8, a developer with a $D-$ log $E$ curve that shows clearly nonlinear behavior. Figure 2 shows (as a function of exposure) the density before bleaching for different $K$ values.

Table 2 presents the values of $m$ and $\xi$ that we used. For each set of plates we adjusted parameters $m$ and $\xi$ so as to have the best agreement between the theoretical curve and the experimental data on $\Delta n$, taking into account that $K$ is different for each set of plates. The values of $m$ and $\xi$ obtained are compatible with the different values of $\mu, K$, and $\beta$ obtained from the experimental data.

Figure 3 shows the behavior of the nonlinear model for the different values of $K$ used in our experiments. As can be seen, when the exposure time increases, so does the SNR. The SNR value also increases as $K$ increases. In Fig. 4 we show the results we obtained from the measurement of diffraction efficiency, $\eta$ [Fig. 4(a)], as the values of the SNR [Fig. 4(b)] of the linear model by Upatnieks and Leonard, by using Eqs. (11) and (14) in Ref. 1:

$$
\mathrm{SNR}=\frac{K}{\left(\sin ^{-1} \sqrt{\eta}\right)^{2}} .
$$

In Fig. 5 we see the results of the comparison we made between the theoretical results of both models and the experimental data. As we can see, for low values of $K$ [Figs. 5(a) and 5(b)] the linear model clearly diverges from the experimental results, while

Table 1. Processing Schedule and Bleach Bath Formula

$\begin{array}{lc}\text { Step Procedure }^{a} & \text { Time } \\ \text { (1) D-8 developer } & 3 \mathrm{~min} \\ \text { (2) Rinse in water } & 30 \mathrm{~s} \\ \text { (3) Fix F-24 } & 5 \mathrm{~min} \\ \text { (4) Wash } & 10 \mathrm{~min} \\ \text { (5) Bleach (for formula, see below) } & 1-5 \mathrm{~min}\end{array}$

(6) Dry

Bleach Formula

Amount

Potassium bromide ( $\mathrm{KBr}$ )

Potassium ferricyanide $\left[\mathrm{K}_{3} \mathrm{Fe}(\mathrm{CN})_{6}\right]$

$7 \mathrm{~g}$

Distilled water to make

$8 \mathrm{~g}$

$1 \mathrm{~L}$

${ }^{a}$ All solutions to $20^{\circ} \mathrm{C}$. 


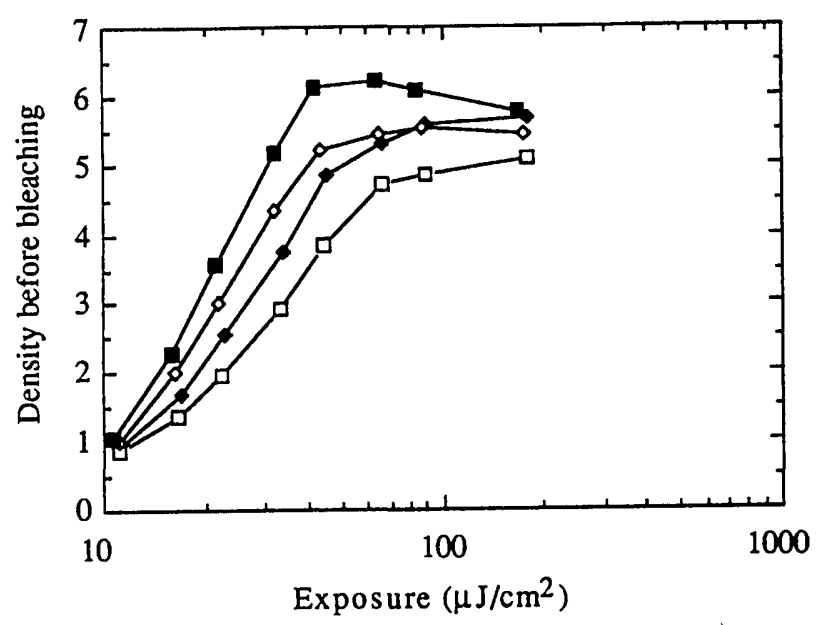

Fig. 2. Density before bleaching, as a function of exposure for $K=$ 1 (hollow squares), $K=5$ (filled diamonds), $K=10$ (hollow diamonds), and $K=25$ (filled squares).

our proposed model comes much closer to the actual experimental data. However, as $K$ increases, the linear model comes closer to the behavior of the experimental values [Figs. 5(c) and $5(\mathrm{~d})$ ]. This is because when $K$ increases, the process becomes more linear even though the model proposed in this paper clearly describes the SNR function more accurately, especially in the nonlinear zones of the $D-\log E$ curve at high exposures.

We should mention here that this study considers only intermodulation noise that is stored as a variation in the refraction index; we do not consider other noise sources such as scattering or noise gratings or the influence of the effects of volume that were pointed out earlier. ${ }^{5}$ Furthermore, intermodulation noise can be stored as thickness variation, and this storage method results in higher levels of noise in the linear zone. In order to isolate the intermodulation noise due to storage of the index variation, we sealed the photographic plates in such a way as to eliminate thickness variations. Figure 6 shows the results of the measurements of the SNR when the plates are sealed, as compared with when they are not sealed, obtained by our nonlinear model. As we can see, in this case the model predicts the experimental results obtained more accurately because in this case we analyzed only noise due to index variation. Experimental results at high exposures correspond better to those obtained in the nonlinear model.

As we have shown, by considering the nonlinear behavior of the recording material in the case of diffuse-object holograms, we achieved a better agree-

Table 2. Values of $K, m$, and $\xi$ Used in the Evaluation of the Signal-to-Noise Ratio

\begin{tabular}{rll}
\hline$K$ & \multicolumn{1}{c}{$m$} & \multicolumn{1}{c}{$\xi$} \\
\hline 1 & 0.5 & 0.12 \\
5 & 0.27 & 0.065 \\
10 & 0.215 & 0.055 \\
25 & 0.13 & 0.042 \\
\hline
\end{tabular}

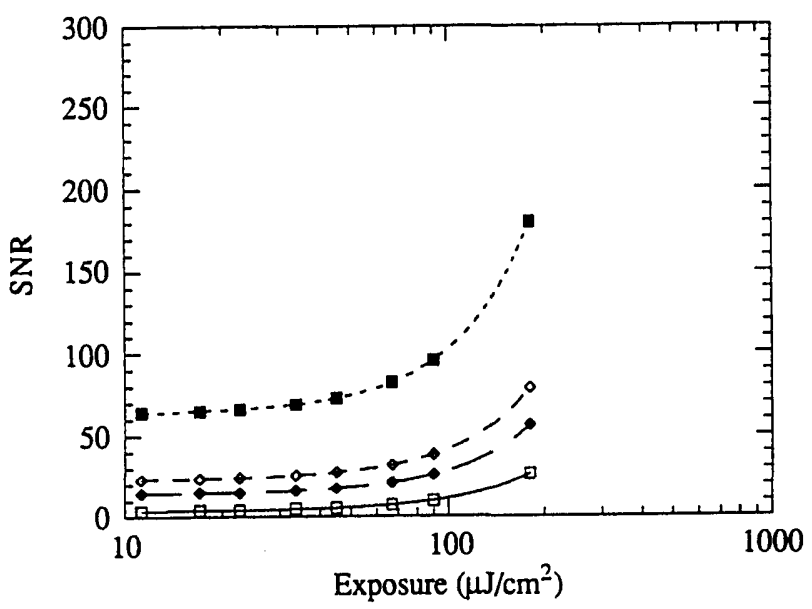

Fig. 3. SNR as a function of exposure for $K=1$ (hollow squares), $K=5$ (filled diamonds), $K=10$ (hollow diamonds), and $K=25$ (filled squares), obtained by nonlinear approximation.

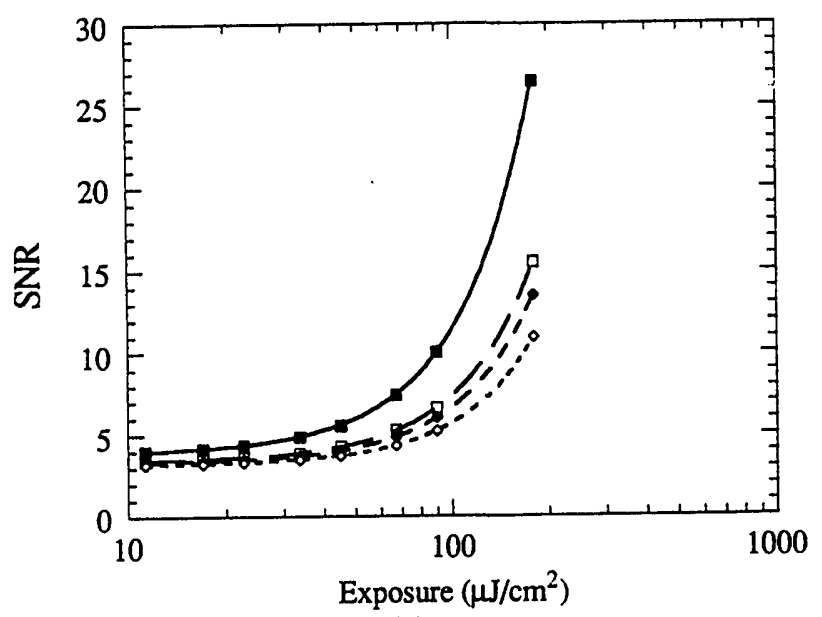

(a)

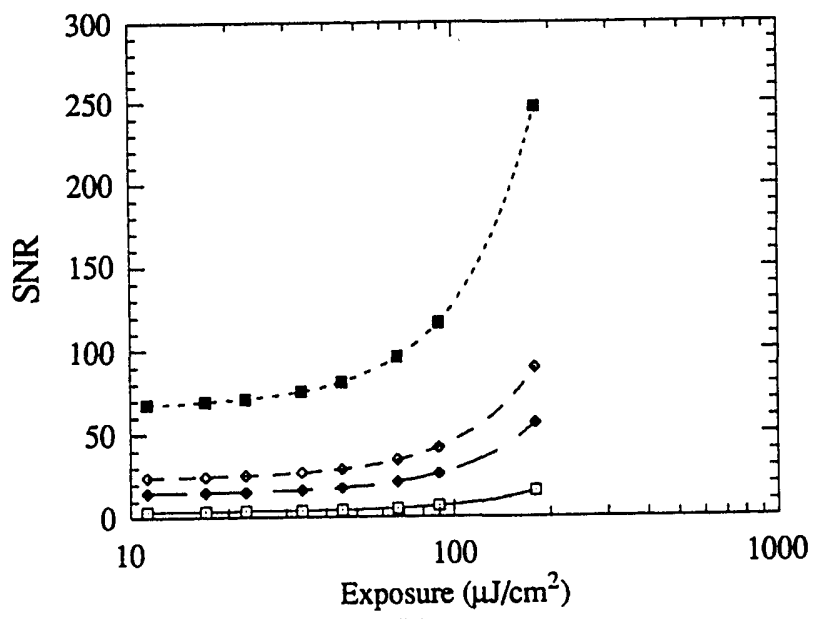

(b)

Fig. 4. (a) SNR as a function of exposure for $K=1$ (filled diamonds), $K=5$ (hollow squares), $K=10$ (hollow diamonds), and $K=25$ (filled squares). (b) SNR as a function of exposure for $K=$ 1 (filled diamonds), $K=5$ (hollow squares); $K=10$ (hollow diamonds), and $K=25$ (filled squares). Both graphs are obtained by linear approximation. 


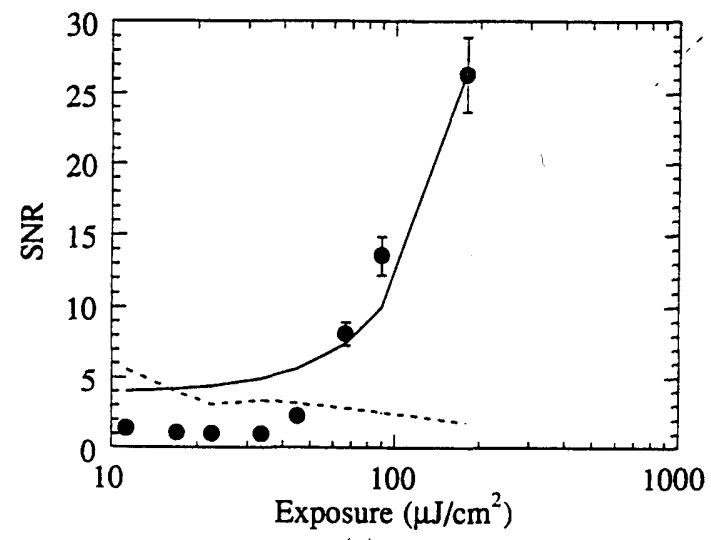

(a)

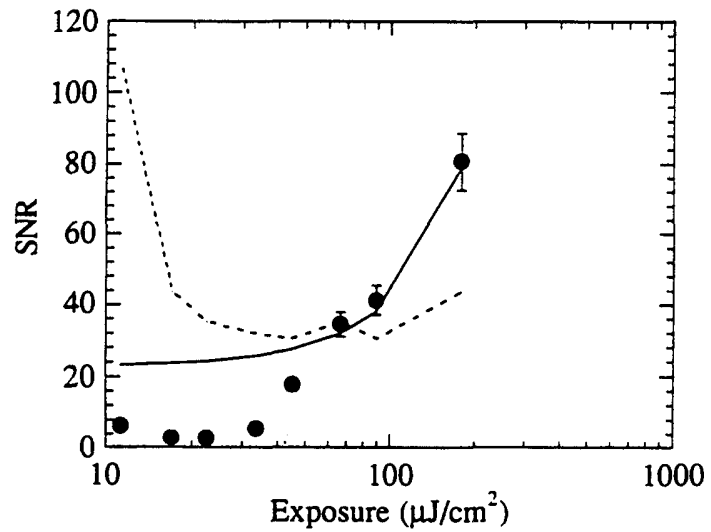

(c)

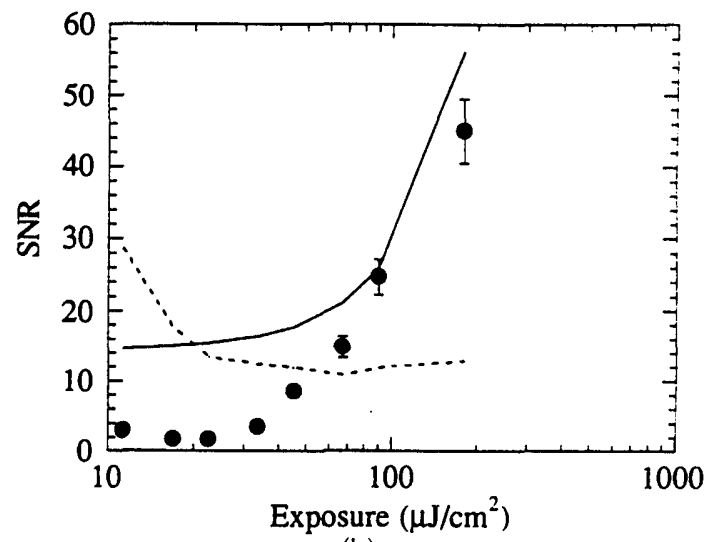

(b)

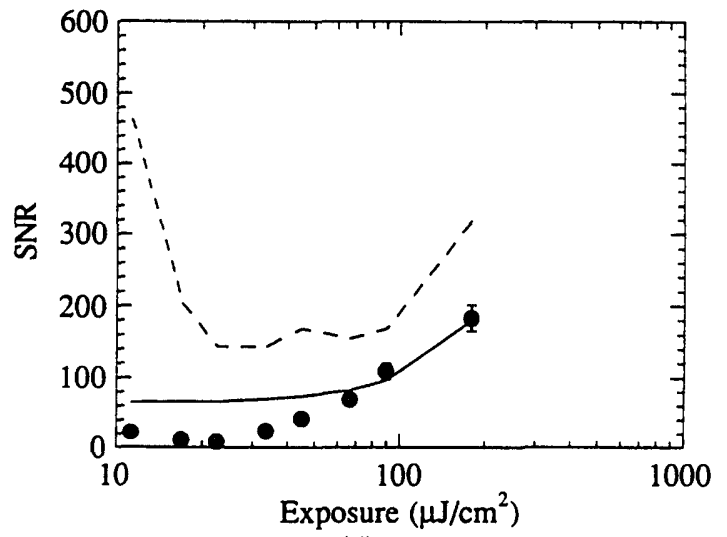

(d)

Fig. 5. Comparison of models used to determine SNR as a function of exposure for (a) $K=1$, (b) $K=5$, (c) $K=10$, and (d) $K=$ 25: experimental data (filled circles), linear model (dashed curves), and nonlinear model (solid curves).

ment between theoretical predictions and experimental results. When the beam ratio $K$ is low, there is agreement between the nonlinear model and the experimental results, but when constant $K=25$, we can observe that the nonlinear model and the experimental results do not agree because in this case the process is linear.

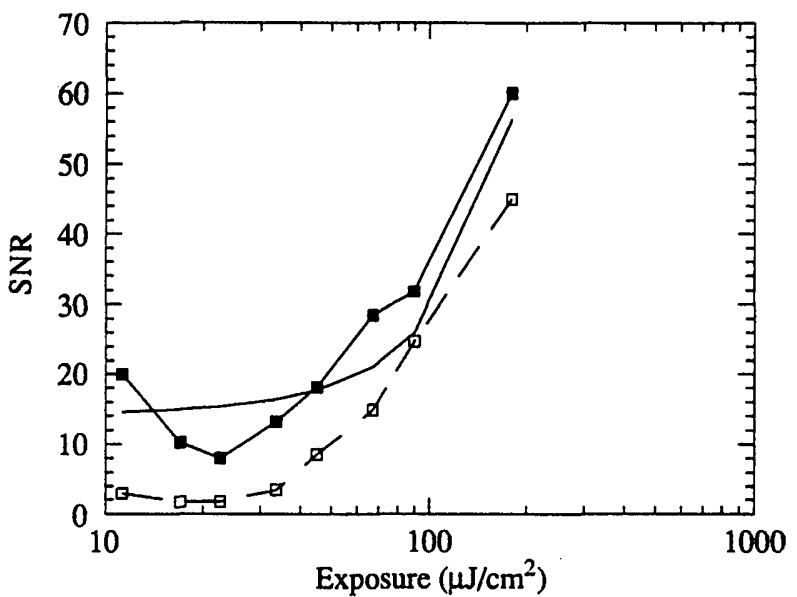

Fig. 6. SNR as a function of exposure for $K=5$ : index-matching plates (filled squares), non-index-matching plates (hollow squares), and nonlinear model (solid curve).

\section{Conclusions}

The SNR's of volume diffuse-object holograms recorded in bleached emulsions have been studied. A nonlinear theoretical model has been developed to explain the nonlinearities in these holograms, and a good agreement between this theory and the experimental results has been found. A discrepancy in the SNR's of the holograms has been attributed to the intermodulation noise (Fig. 6). Finally, we should point out once more that other noise sources have not been considered in this model and that we have included only the influence of intermodulation noise stored as refraction-index variation. These results are important given that they are related to cross talk in holographic interconnection systems used in optical computing, in which the models proposed up to now are linear. ${ }^{6}$

Part of this work was supported by the Direcció General d'Ensenyaments Universitaris i Investigació de la Generalitat Valenciana, Spain (project GV1165/93).

\section{References}

1. J. Upatnieks and C. D. Leonard, "Efficiency and image contrast of dielectric holograms," J. Opt. Soc. Am. 60, 297-305 (1970).

2. R. K. Kostuk, "Comparison of models for multiplexed holograms," Appl. Opt. 28, 771-777 (1989). 
3. A. Fimia, M. Pardo, and J. A. Quintana, "Improvement of image quality in bleached holograms," Appl. Opt. 21, 3412-3413 (1982).

4. R. L. van Renesse and N. J. van der Zwaal, "Refractive index and thickness variations of the photographic emulsion," Opt. Laser Technol. (February 1971), 41-44.
5. A. Fimia, L. Carretero, and R. Fuentes, "Volume influence on intermodulation noise of dielectric diffuse-object holograms," Appl. Opt. 31, 2408-2049 (1992).

6. P. Asthana, G. P. Nordin, A. R. Tanguay, Jr., and B. K. Jenkins, "Analysis of weighted fan-out/fan-in volume holographic optical interconnections," Appl. Opt. 32, 1441-1469 (1993). 